\title{
Spectroscopic observations of eccentric eclipsing binary systems
}

\author{
E. Kiran, V. Bakiş and H. Bakiş \\ Department of Space Sciences and Technologies, Faculty of Science, Akdeniz \\ University,07058, Antalya,Turkey,(E-mail: evrimkiran@akdeniz.edu.tr)
}

Received: November 8, 2019; Accepted: February 25, 2020

\begin{abstract}
Eccentric eclipsing binaries (EEBs) are detached binary stars. Many EEBs show apsidal motion. EEB fundamental physical parameters allow us to test stellar evolution models and to estimate the internal stellar structure constants through apsidal motion analysis. We started a programme to study EEBs at the UBT60 telescope of Akdeniz University located at TÜBİTAK National Observatory. In this paper, we present spectroscopic orbital parameters of some selected EEBs.
\end{abstract}

Key words: stars: binaries: detached - stars: binaries: eclipsing - techniques: spectroscopic

\section{Introduction}

Eclipsing binary systems are important objects that help us understand stellar and binary evolution in astrophysics. Eccentric eclipsing binaries (EEBs) are precious astrophysical laboratories to study apsidal motion and test general relativity. The rotation of the eccentric orbit is known as apsidal motion. The study of the apsidal motion provides information on the internal structure constants of the components. The internal structure constants and precise absolute parameters of the components can be used to test evolutionary models. The physical properties of the components of the systems can be derived from the study of spectroscopic and photometric observations. Light curves of binaries with long orbital periods can be obtained from published photometric catalogues. However, it is difficult to obtain complete radial velocity curves of eclipsing binary systems with long orbital periods. This study is in part a continuation of our programme of spectroscopic observations of long-period eclipsing binary systems. We collected the spectra of two early type eccentric eclipsing binaries, V839 Cep and V850 Cep, and obtained their spectroscopic orbital parameters.

The light elements of V839 Cep (GSC 3964-0741) were given by Otero et al. $(2006)$ as $T_{0}$ (HJD) $=2451448.645$ and period $P=9.9634 \mathrm{~d}$. Detailed apsidal motion studies were performed by Zasche et al. (2018) and Volkov et al. (2019). Here we derive absolute parameters of the system. 
V850 Cep (GSC 04257-00906) was listed as an eccentric binary system by Otero et al. (2006) who list ephemeris parameters $T_{0}$ (HJD) $=2451475.710$ and period $P=12.922 \mathrm{~d}$.

\section{Observations and data reduction}

Observations were carried with the Akdeniz University 0.6-m telescope (UBT60) located at TÜBİTAK National Observatory. The eShel spectrograph equipped with a QSI632s CCD camera (pixel size $6.8 \mu \mathrm{m}$, gain $0.9 \mathrm{e}^{-} / \mathrm{ADU}$, readout noise $7 \mathrm{e}^{-} \mathrm{i}$ was used to collect the spectral data. The eShel spectrograph, which has a resolution power of 12000 and covers a $4045-8100 \AA$ wavelength range in 27 orders, is connected to the telescope with fiber optics.

In each observation night, we obtained bias, dark frames, flat spectra from a Tungsten lamp, and wavelength calibration spectra from a ThAr lamp for the calibration of the spectra. The IRAF ${ }^{1}$ echelle package was used for extracting order apertures, elimination of scattered light through the orders, wavelength calibrations of spectroscopic data and measuring the radial velocities (RVs) of components.

Table 1. The orbital parameters of V839 Cep and V850 Cep.

\begin{tabular}{lcc}
\hline \hline & V839 Cep & V850 Cep \\
\hline$T_{\text {per }}(\mathrm{HJD})$ & $2458371.83872 \pm 0.47141$ & $2457972.65588 \pm 1.05112$ \\
$P$ (days) & 9.9633 (fixed) & 12.9285 (fixed) \\
$\omega($ deg) & $31.58 \pm 15$ & $284 \pm 14$ \\
$e$ & $0.20 \pm 0.04$ & $0.37 \pm 0.15$ \\
$K_{1}\left(\mathrm{~km} \mathrm{~s}^{-1}\right)$ & $80.3 \pm 5.0$ & $80 \pm 15$ \\
$K_{2}\left(\mathrm{~km} \mathrm{~s}^{-1}\right)$ & $79.7 \pm 5.0$ & $84 \pm 17$ \\
$\mathrm{~V}_{\gamma}\left(\mathrm{km} \mathrm{s}^{-1}\right)$ & $-32.2 \pm 3.0$ & $-25.7 \pm 6.1$ \\
$a_{1} \sin i(\mathrm{~km})$ & $108 \pm 9 \times 10^{5}$ & $13.2 \pm 3.4 \times 10^{6}$ \\
$a_{2} \sin i(\mathrm{~km})$ & $107 \pm 9 \times 10^{5}$ & $13.8 \pm 3.7 \times 10^{6}$ \\
$M_{1} \sin ^{3} i\left(\mathrm{M}_{\odot}\right)$ & $1.99 \pm 0.33$ & $2.41 \pm 1.50$ \\
$M_{2} \sin ^{3} i\left(\mathrm{M}_{\odot}\right)$ & $2.00 \pm 0.33$ & $2.31 \pm 1.43$ \\
\hline \hline
\end{tabular}

\section{Preliminary Results}

The RVs were measured by applying Gaussian profiles to the central parts of the $\mathrm{H} \alpha(6563 \AA)$ line. The least-squares method was used to fit the RV equation

\footnotetext{
${ }^{1}$ IRAF is distributed by the National Optical Observatories, operated by the Association of Universities for research in Astronomy, Inc., under cooperative agreement with the National Science Foundation.
} 

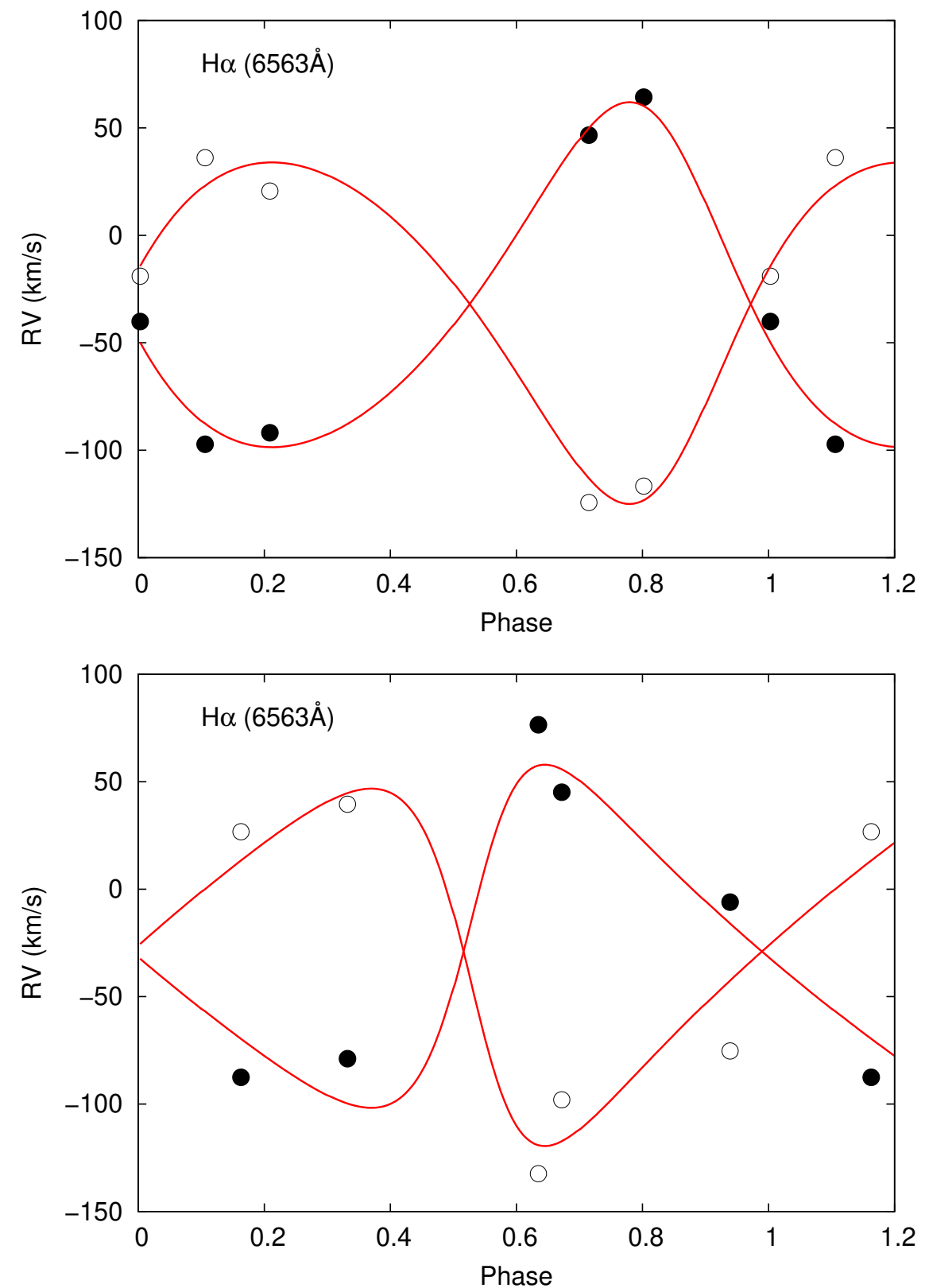

Figure 1. Radial velocities of V839 Cep (upper panel) and V850 Cep (lower panel). 
to the measured RVs. Radial velocity curves (RVCs) and the theoretical orbital fitting can be seen in Fig. 1 for V839 Cep and V850 Cep. Filled and empty dots represent the RVs of the primary and secondary component, respectively. Dashed red lines are synthetic curves fitted to the RVs. We used the light elements parameters given by Kreiner (2004) as initial parameters and kept periods fixed during the solutions. The results of the RVC solutions for the systems are given in Table 1.

Orbital parameters for V839 Cep and V850 Cep are presented based on new spectroscopic observations. The theoretical curves fit the RVs well for V839 Cep and orbital parameters of the system were obtained. Simultaneous RV and light curve solutions allow us to derive the absolute parameters of the components. The large error values of the orbital parameters of V850 Cep are due to the small number of RV data points. More spectroscopic observations are needed for this system to obtain more accurate orbital parameters.

Acknowledgements. This study was supported by the TÜBITAK 1002-Short Term R\&D Funding Program.

\section{References}

Kreiner, J. M., Up-to-Date Linear Elements of Eclipsing Binaries. 2004, Acta Astron., 54, 207

Otero, S. A., Wils, P., Hoogeveen, G., \& Dubovsky, P. A., 50 New Eccentric Eclipsing Binaries Found in the ASAS, Hipparcos and NSVS Databases. 2006, Information Bulletin on Variable Stars, 5681, 1

Volkov, I. M., Bagaev, L. A., Kravtsova, A. S., \& Chochol, D., V839 Cep - a new massive eclipsing variable with apsidal motion in the field of Trumpler 37. 2019, Contributions of the Astronomical Observatory Skalnate Pleso, 49, 434

Zasche, P., Wolf, M., Uhlař, R., et al., The first study of 54 new eccentric eclipsing binaries in our Galaxy. 2018, Astron. Astrophys., 619, A85 